\title{
Universality and itinerant ferromagnetism in rotating strongly interacting Fermi gases
}

\author{
B. C. Mulkerin, C. J. Bradly, H. M. Quiney, and A. M. Martin \\ School of Physics, University of Melbourne, Victoria 3010, Australia \\ (Received 17 July 2012; revised manuscript received 31 July 2012; published 30 November 2012)
}

\begin{abstract}
We analytically determine the properties of three interacting fermions in a harmonic trap subject to an external rotation. Many-body thermodynamic quantities such as the entropy and energy are calculated from the third-order quantum virial expansion. As the external rotation is increased, the regime over which the virial expansion is valid is extended to lower temperatures. By parameterizing the solutions in the rotating frame we find that the energy and entropy are universal for all rotations in the strongly interacting regime. Additionally, we find that rotation suppresses the onset of itinerant ferromagnetism in strongly interacting repulsive three-body systems.
\end{abstract}

DOI: 10.1103/PhysRevA.86.053631

PACS number(s): 67.85.-d, 03.75.Hh, 03.75.Ss, 05.30.Fk

Strongly interacting Fermi gases are thought to have universal thermodynamic behavior [1-6]. This regime has been accessed in experiment [7-16] and may provide insight into the properties of neutron stars and more conventional solid-state physics [17-20]. It is the presence of broad Feshbach resonances in two-component atomic Fermi gases that has made it possible to access the strongly interacting regime. In this regime the difficulties in developing manybody theories for these systems has motivated the study of exact solutions to few-body problems as a means to gain insight into the many-body problem [21-29]. Of particular interest has been the prediction $[6,27,30,31]$ and observation $[15,16]$ of universality in the strongly interacting regime of thermodynamic quantities, such as the energy and entropy. To date such calculations have been restricted to gases in nonrotating, spherically symmetric harmonic traps.

In this work we consider the properties of a strongly interacting gas in a rotating trap. In particular, we solve the two- [32] and three-body problems and calculate the virial expansion of the thermodynamic potential to third order, enabling the calculation of thermodynamic quantities. We find that the temperatures above which the virial expansion is valid decrease as external rotation increases. Additionally, we show in the thermodynamic limit that the second- and third-order virial coefficients are universal with respect to an external rotation and trapping frequencies. From this we find that the many-body thermodynamic quantities such as energy and entropy are universal with respect to rotation through a simple rescaling of the Fermi energy.

In addition to these thermodynamic results, we also examine the interplay between rotation and the emergence of itinerant ferromagnetism in strongly interacting ultracold Fermi gases. In the original work of Stoner [33] it was proposed via a mean-field theory that a repulsive Fermi gas will always exhibit a ferromagnetic phase. Experimental work suggests the observation of a ferromagnetic phase for a strongly interacting Fermi gas, with repulsive interactions [34], in the absence of rotation. However, this is contradicted by the recent experimental work of Sanner et al. [35], which suggest the rapid formation of molecules inhibits the formation of a ferromagnetic phase. Current theoretical work, Monte Carlo simulations, and Tan relations [27,36-38] are also contradictory. In this work we show that for the three-body problem, rotation suppresses the emergence of itinerant ferromagnetism. Additionally, in the thermodynamic limit we find that itinerant ferromagnetism is suppressed for temperatures $T>10^{-7} T_{\mathrm{F}}$ as the rotation frequency approaches the trapping frequency.

Our starting point is the wave function $\psi\left(\mathbf{r}_{1}, \mathbf{r}_{2}, \ldots\right)$ of $N$ particles interacting in the $s$-wave channel at low energies. This satisfies the Bethe-Peierls boundary conditions

$$
\lim _{r_{i j} \rightarrow 0} \partial\left(r_{i j} \psi\right) / \partial r_{i j}=-r_{i j} \psi / a,
$$

where the interaction is parametrized by the scattering length $a$, and $r_{i j}=\left|\mathbf{r}_{i}-\mathbf{r}_{j}\right|$ is the separation of opposite-spin fermions. Away from $r_{i j}=0$, the wave function of $N$ particles in a spherically symmetric rotating harmonic trap satisfies the noninteracting Schrödinger equation,

$$
\sum_{i=1}^{N}\left[-\frac{\hbar^{2}}{2 \mu} \nabla_{i}^{2}+\frac{1}{2} \mu \omega^{2} \mathbf{r}_{i}^{2}+i \hbar \Omega_{z} \partial_{\phi_{i}}\right] \psi=E \psi,
$$

where $\mathbf{r}_{i}$ and $\mu$ are the position and mass of each particle, and $\omega$ and $\Omega_{z}$ are the trapping and rotation frequencies, the latter assumed to be defined about the $z$ axis.

The center-of-mass Hamiltonian can be decoupled from Eq. (2) and defines the rotating harmonic motion of a particle of mass $M=N \mu$ with energy spectrum

$$
E_{\mathrm{cm}}=(2 n+l+3 / 2) \hbar \omega+m \hbar \Omega_{z},
$$

where $n, l, m$ label the usual harmonic oscillator eigenstates $R_{n l} Y_{l}^{m}$. The relative energy, $E_{\text {rel }}=E-E_{\text {c.m. }}$, incorporates the effects of the contact interaction but not the effects of the external rotation, since only $s$-wave states may interact. For two opposite-spin fermions the wave function in relative coordinates that satisfies the Bethe-Peierls boundary condition Eq. (1) is [21] $\psi_{2 b}^{\mathrm{rel}}(\mathbf{r} ; v) \propto \Gamma(-v) U\left(-v, 3 / 2, r^{2} / d^{2}\right) \exp \left(-r^{2} / d^{2}\right)$, where $U$ is the confluent hypergeometric function of the second kind. A pseudoquantum number $v$ parametrizes the relative energy $E_{\mathrm{rel}}=(2 v+3 / 2) \hbar \omega$ and satisfies the relation $2 \Gamma(-v) / \Gamma(-v-1 / 2)=d / a$ for harmonic oscillator length $d=\sqrt{\hbar /(\mu \omega)}$. In particular, for the unitary limit where $a \rightarrow \pm \infty$, the relative energy spectrum simplifies to $E_{\mathrm{rel}}=$ $(2 n+1 / 2) \hbar \omega$, where $n$ is any non-negative integer.

To find the energy spectrum of three interacting fermions in a rotating trap, we consider the configuration of two spin up fermions and one spin down, $\uparrow \downarrow \uparrow$, where two opposite spin particles interact at a point and form a pair, and the third moves relative to the pair. We define the center-of-mass coordinate of 
the three particles as $\mathbf{R}=\left(\mathbf{r}_{1}+\mathbf{r}_{2}+\mathbf{r}_{3}\right) / 3$, the relative coordinate between the interacting pair $\mathbf{r}=\mathbf{r}_{1}-\mathbf{r}_{2}$, and the relative coordinate between the third noninteracting particle and the center-of-mass of the pair as $\rho=(2 / \sqrt{3})\left[\mathbf{r}_{3}-\left(\mathbf{r}_{1}+\mathbf{r}_{2}\right) / 2\right]$. In this Jacobi coordinate system the center-of-mass Hamiltonian decouples from the relative Hamiltonian

$$
H_{\mathrm{rel}}=-\frac{\hbar^{2}}{\mu}\left(\nabla_{\mathbf{r}}^{2}+\nabla_{\rho}^{2}\right)+\frac{1}{4} \mu \omega^{2}\left(\mathbf{r}^{2}+\rho^{2}\right)-i \hbar \Omega_{z} \partial_{\phi_{\rho}},
$$

where $\mu / 2$ is the reduced mass of the interacting pair. Like the two-body system, the angular momentum vanishes in the interacting pair, but the third fermion can rotate around the pair and be affected by the external rotation, $\Omega_{z}$. This couples higher-order angular momentum states to lower energies in the system. In order to solve the relative Hamiltonian (4) we take

$$
\Psi_{3 b}^{\mathrm{rel}}(\mathbf{r}, \rho)=\left(1-P_{13}\right) \sum_{n=0}^{\infty} c_{n} \psi_{2 b}^{\mathrm{rel}}\left(\mathbf{r} ; v_{n l m}\right) R_{n m}(\rho) Y_{l}^{m}(\hat{\rho})
$$

as an ansatz for the wave function, where $P_{13}$ is an operator that exchanges the spin $\uparrow$ particles. The eigenenergies of this system are

$$
E_{\mathrm{rel}}=\left[\left(2 n+l+\frac{3}{2}\right)+\left(2 v_{n l m}+\frac{3}{2}\right)\right] \hbar \omega+m \hbar \Omega_{z} .
$$

The presence of the rotational term $m \hbar \Omega_{z}$ in the eigenenergy spectrum shifts the nonrotating energy spectrum found in [26]. To solve the relative energy spectrum using the wave-function ansatz Eq. (5), we utilize the Bethe-Peierls boundary condition Eq. (1) and choose a set of quantum numbers $n l m$ and energy $E_{\text {rel }}$ to solve for a particular $v_{n l m}$ and scattering length $a$ [26]. The energy spectrum of three interacting fermions is shown in Fig. 1 for a rotation of $\Omega_{z}=0.9 \omega$, a relative angular momentum of $l=2$, and axial angular momentum of $m=-2,0,2$. We see the energy levels for the three interacting fermions shift for each $m$ quantum number, lifting the $(2 l+1)$ fold degeneracy.

To calculate the virial coefficients and hence thermodynamic properties in the unitary regime it is simpler to generalize the method of Werner and Castin [22,23] using hyperspherical coordinates to obtain the relative energy spectrum

$$
E_{\mathrm{rel}}=\left(2 q+s_{n l}+1\right) \hbar \omega+m \hbar \Omega_{z},
$$

where $q$ is a non-negative integer and $s_{n l}$ are positive and determined by the eigenvalues of

$$
-\varphi^{\prime \prime}(\alpha)+\frac{l(l+1)}{\cos ^{2}(\alpha)} \varphi(\alpha)=s_{n l}^{2} \varphi(\alpha)
$$

where $\alpha=\arctan (r / \rho)$ is the hyperangle. The eigenvalues $s_{n l}$ are determined from the Bethe-Peierls boundary condition (1), which in hyperspherical coordinates reads $\varphi^{\prime}(0)-(-1)^{l} 4 / \sqrt{3} \varphi(\pi / 3)=0$. The procedure for solving for values of $s_{n l}$ using the general solutions to the hyperangle equation is given in [27].

To obtain the repulsive spectrum we omit the $s_{0 l}$ energy levels, which are the lowest in each $l$ subspace. In doing this we assume that the formation of molecules is inhibited, which may not be the case when the scattering rate is changed nonadiabatically [35]. The lowest energy in the repulsive regime is then the relative energy, which is dependent upon
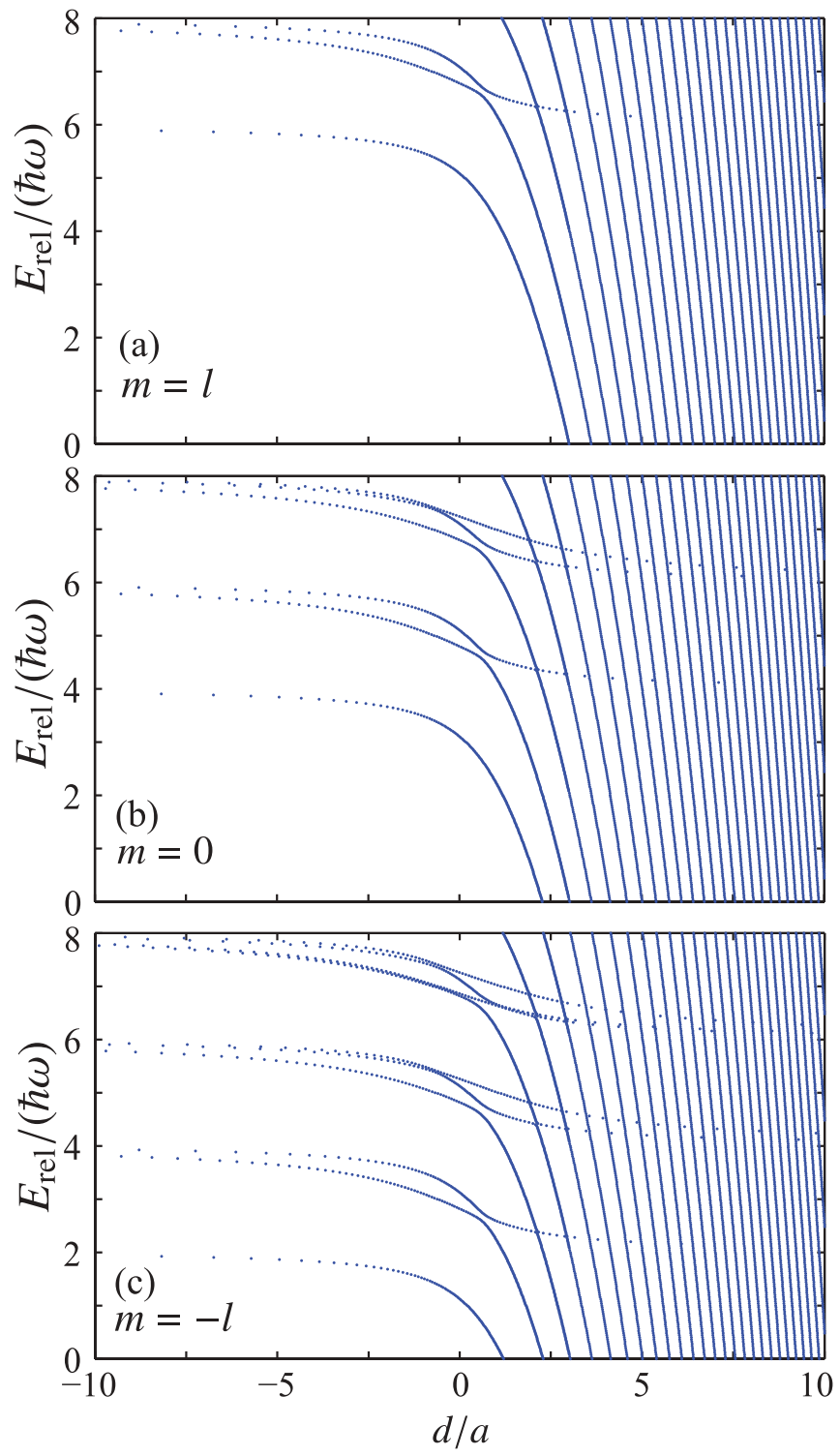

FIG. 1. (Color online) Energy spectrum of three interacting fermions with $l=2$ and $m=-2,0$, and 2 for plots (a), (b), and (c), respectively, with a rotation of $\Omega_{z}=0.9 \omega$. We can see the shifting of the energy spectrum with the rotation included; in particular, we can see the number of lower-energy states increase for $m=-2$.

the rotation $\Omega_{z}$, plus the center-of-mass energy

$$
E_{g s}^{\uparrow \downarrow \uparrow}=\left(s_{n l}+1\right) \hbar \omega+m \hbar \Omega_{z}+1.5 \hbar \omega .
$$

This can be compared to the energy of three polarized fermions, which is the sum of the three lowest allowed energies:

$$
E_{g s}^{\uparrow \uparrow}=1.5 \hbar \omega+2.5 \hbar \omega+\left[1.5 \hbar \omega+\left(\omega-\Omega_{z}\right) \hbar\right] .
$$

Comparing Eqs. (9) and (10) it is possible to find a critical rotation $\Omega_{c}$, for which the ground-state energy of three repulsively interacting fermions becomes lower than three noninteracting polarized fermions. This is a rotation for which the three noninteracting fermions are unstable with respect to the three-interacting-fermion system. Choosing the lowest relative energy level in the $l=2$ subspace, $s_{12} \simeq 4.80$, and a magnetic quantum number $m=-l$, we find a critical 
rotation of $\Omega_{c} \simeq 0.8 \omega$. Since the ground-state energy of three repulsively interacting fermions can be controlled by varying an external rotation, this indicates that an itinerant ferromagnetic transition, in the three-body system, only occurs for a rotation $\Omega_{z}<\Omega_{c}$.

The two- [32] and three-body solutions can now be used to calculate the many-body properties of strongly interacting rotating Fermi gases. This is done via a quantum virial expansion of the grand thermodynamic potential, $\Phi=-k_{B} T \ln \mathcal{Z}$, in terms of the fugacity $z$ :

$$
\begin{aligned}
\Phi= & -k_{B} T Q_{1} \frac{1}{2} \int_{0}^{\infty} d \epsilon \epsilon^{2} \ln \left(1+z e^{-\epsilon}\right) \\
& -k_{B} T Q_{1}\left(z+\Delta b_{2} z^{2}+\Delta b_{3} z^{3}+\cdots\right)
\end{aligned}
$$

where

$$
\begin{gathered}
\Delta b_{2}=\Delta Q_{2} / Q_{1}, \\
\Delta b_{3}=\Delta Q_{3} / Q_{1}-\Delta Q_{2},
\end{gathered}
$$

and $\Delta Q_{N}=Q_{N}-Q_{N}^{(0)}$ with $Q_{N}=\operatorname{Tr}\left[\exp \left(-\mathcal{H}_{N} / k_{B} T\right)\right]$ and $Q_{N}^{(0)}$ the noninteracting cluster function [39]. To first order for dimensionless rotation $\xi=\Omega_{z} / \omega, Q_{1}$ is given by

$$
Q_{1}=\left(\frac{k_{\mathrm{B}} T}{\hbar \omega}\right)^{3} \frac{1}{1-\xi^{2}}+\cdots .
$$

Following [32], the second-order virial coefficient for a rotating trapped gas in the high-temperature limit is

$$
\begin{aligned}
\Delta b_{2}^{\text {att }} & =\frac{1}{4}-\frac{\tilde{\omega}^{2}}{32}+\cdots, \\
\Delta b_{2}^{\text {rep }} & =-\frac{1}{4}+\frac{\tilde{\omega}}{4}+\cdots,
\end{aligned}
$$

where $\tilde{\omega}=\hbar \omega / k_{\mathrm{B}} T$ is the reduced trapping frequency and $\tilde{\omega} \rightarrow 0$ represents the thermodynamic limit. By extending the work of nonrotating systems in [26] we find that $\Delta b_{3}^{\text {att }}$ is universal for any rotation and given by

$$
\Delta b_{3}^{\text {att }} \simeq-0.06833960+O\left(\tilde{\omega}^{2}\right) .
$$

For a repulsive gas the third-order virial coefficient to lowest order in $\tilde{\omega}$ is also universal,

$$
\Delta b_{3}^{\text {rep }} \simeq 0.34976+O(\tilde{\omega}) .
$$

Despite the rotational dependence of the two- and three-body eigenspectrums, the second- and third-order virial coefficients are independent of rotation in the thermodynamic limit.

We are now able to calculate the total energy $E=-3 \Phi$ and entropy $S=-\partial \Phi / \partial T$ from the thermodynamic potential $\Phi$ of a strongly interacting gas [39]. To determine the thermodynamic potential, the fugacity $z=\exp \left(\mu / k_{\mathrm{B}} T\right)$ must be calculated from the total number of particles $N=-\partial \Phi / \partial \mu$, where $\mu$ is the chemical potential.

In Fig. 2 we plot the energy (a), (b) per particle and entropy (c), (d) per particle as functions of rotation using the virial expansion to third order in the strongly interacting regime. The repulsive (b), (d) and attractive (a), (c) regimes are plotted as functions of reduced temperature $T_{\mathrm{F}}^{0}=E_{\mathrm{F}}^{0} / k_{\mathrm{B}}$, where $E_{\mathrm{F}}^{0}=(3 N)^{1 / 3} \hbar \omega$ is the Fermi energy. The hashed areas in plots (a) and (c) correspond to solutions of the energy and entropy that are unphysical. To quantify the regimes of
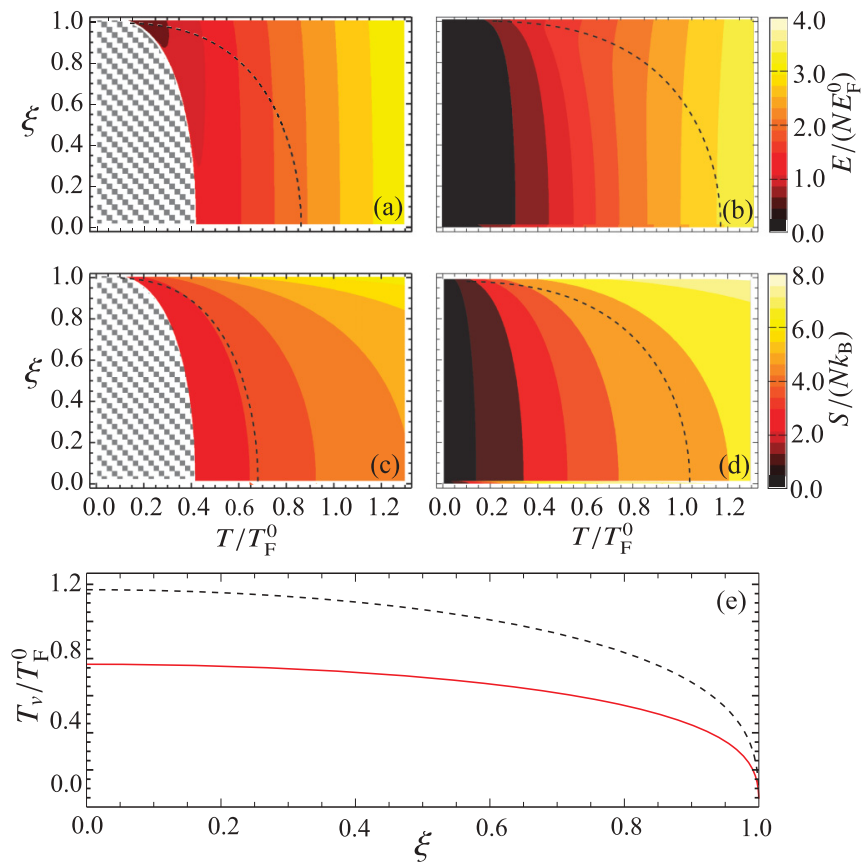

FIG. 2. (Color online) (a), (b) Energy per particle $E /\left(N E_{\mathrm{F}}^{0}\right)$ and (c), (d) entropy per particle $S /\left(N k_{\mathrm{B}}\right)$ for dimensionless rotation $\xi$ as a function of reduced temperature $T / T_{\mathrm{F}}^{0}$, in the strongly (a), (c) attractive and (b), (d) repulsive regimes. For comparison we plot the temperature at which the second- and third-order virial expansions differ by $1 \%$ for a rotation $\xi$, the dashed curve. The hashed region in plots (a) and (c) indicate an unphysical solution to the virial expansion. (e) The temperature $T_{v} / T_{\mathrm{F}}^{0}$, for attractive (solid black curve) and repulsive (dashed red curve), above which the energy as determined by the second- and third-order virial expansions differs by $<1 \%$.

validity, in Fig. 2(e) we plot the temperature $T_{v} / T_{\mathrm{F}}^{0}$ above which the virial expansion is valid as a function of rotation for attractive (solid black curve) and repulsive (dashed red curve) interactions. $T_{v}$ is defined via a comparison of the energy using the second- and third-order virial expansion. Interestingly, we observe that as the external rotation increases the regime of validity extends to lower temperatures for both attractive and repulsive interactions. In the limit $\xi \rightarrow 1$ these results suggest that the virial expansion to second order is valid to extremely low temperatures, i.e., $T_{v} \rightarrow 0$. As the rotation is changed, for a fixed temperature, the energy (a), (b) and entropy (c), (d) change. Hence, the thermodynamic quantities appear not to be universal with respect to rotation.

Equations (9) and (10) show that for a three-body system the ferromagnetic state is suppressed in the presence of strong repulsive interactions for $\Omega_{z} \gtrsim 0.8 \omega$. Figure 2(b) plots the total energy in the strongly repulsive many-body regime. Figure 2(e) shows that as $\xi \rightarrow 1$ the validity of the solutions extends to $T \rightarrow 0$. In this regime, by comparing the energy of the strongly interacting gas with the equivalent noninteracting polarized gas, we find that the itinerant ferromagnetic phase is suppressed for $T / T_{\mathrm{F}}^{0} \gtrsim 10^{-7}{ }^{1}$

\footnotetext{
${ }^{1}$ Due to numerical instabilities it is not possible to set $\xi=1$. Hence the limit $\xi \rightarrow 1$ is evaluated at $\xi=1-10^{-7}$, for which the virial expansion is valid for $T / T_{\mathrm{F}}^{0} \gtrsim 10^{-7}$.
} 

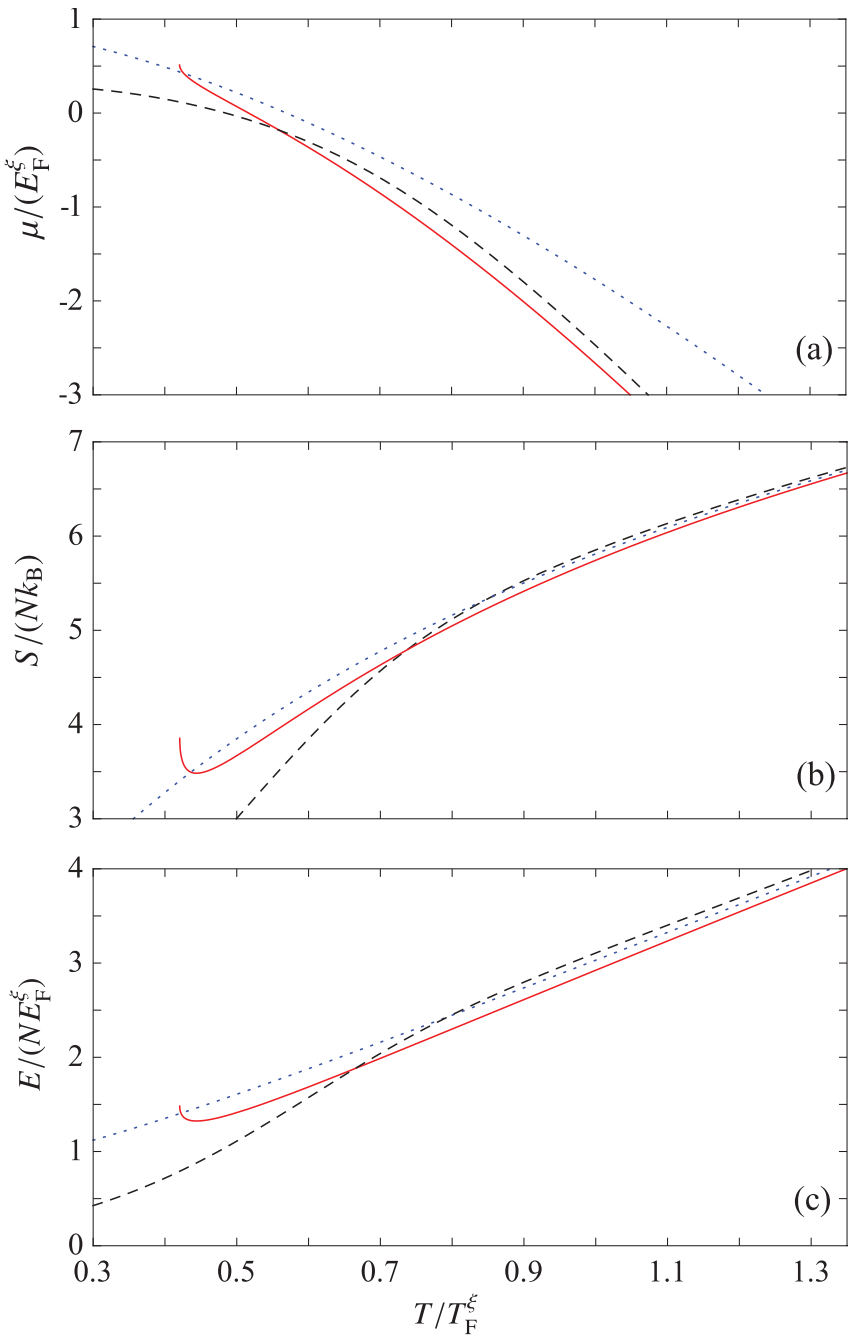

FIG. 3. (Color online) Universal curves: chemical potential (a), entropy per particle (b), and energy per particle (c) of a strongly attractive (solid red curve), repulsive (dashed black curve), and ideal Fermi gas (dotted blue curve) for dimensionless rotation $0 \leqslant \xi \leqslant 1$ as a function of reduced temperature $T / T_{\mathrm{F}}^{\xi}$.

Figures 2(a)-2(d) demonstrate that for a given rotation frequency $\xi$ there is a universal dependence of $E /\left(N E_{\mathrm{F}}^{0}\right)$ and $S /\left(N k_{\mathrm{B}}\right)$ as a function of $T_{\mathrm{F}}^{0}$ with respect to particle number and trapping frequency. However, through a simple rescaling of the Fermi energy [temperature] of the form $E_{\mathrm{F}}^{\xi}=E_{\mathrm{F}}^{0}\left(1-\xi^{2}\right)^{-1 / 3}\left[T_{\mathrm{F}}^{\xi}=T_{\mathrm{F}}^{0}\left(1-\xi^{2}\right)^{-1 / 3}\right]$ it is possible to exactly remove the rotational dependence observed in
Figs. 2(a)-2(d). This generalized universality arises from the fact that under this rescaling the functional dependence of $\xi$ is removed from both the single-particle cluster function $Q_{1}$ [Eq. (14)] and the chemical potential. In conjunction with the fact that the second- [Eqs. (15) and (16)] and third-order [Eqs. (17) and (18)] virial coefficients are independent of rotation in the thermodynamic limit, the thermodynamic potential is analytically independent of rotation. To emphasize the exact universal nature of the chemical potential with respect to rotation, $\mu / E_{\mathrm{F}}^{\xi}$ is plotted in Fig. 3(a) as a function of $T / T_{\mathrm{F}}^{\xi}$ for $0 \leqslant \xi \leqslant 1$ in the strongly attractive (solid red curve), repulsive (dashed black curve), and ideal (dotted blue curve) regimes. As a direct consequence the rescaled energy $E /\left(N E_{\mathrm{F}}^{\xi}\right)$ and entropy $S /\left(N k_{\mathrm{B}}\right)$ are exact universal functions of $T / T_{\mathrm{F}}^{\xi}$ with respect to rotation and trapping frequency. This property is demonstrated in Figs. 3(b) and 3(c), which plot $E /\left(N E_{\mathrm{F}}^{\xi}\right)$ (b) and $S /\left(N k_{\mathrm{B}}\right)$ (c) as a function of $T / T_{\mathrm{F}}^{\xi}$ for $0 \leqslant \xi \leqslant 1$ in the strongly attractive (solid red curve), repulsive (dashed black curve), and ideal (dotted blue curve) regimes.

In conclusion, we have examined the problem of three ultracold fermions in a harmonic trap subject to an external rotation. For the three-body problem we have demonstrated that rotation suppresses the transition to a ferromagnetic state. Explicitly we have shown that the three-body ferromagnetic state has a higher energy than the strongly interacting repulsive state for $\Omega_{z} \gtrsim 0.8 \omega$ and is, consequently, unstable. Additionally, from the three-body solutions and the use of previous two-body results [32], we have calculated the many-body equations of state using the virial expansion to third order. We find that the introduction of rotation broadens the validity of the virial expansion to lower temperatures, and as the rotation frequency approaches the trapping frequency the validity of the virial expansion extends to zero temperature. An obvious and as-yet-unanswered question is, does the validity of the virial expansion extend to zero temperature as the rotation frequency approaches the trapping frequency for higher-order virial expansions? Despite the rotational dependence of the two- and three-body eigenspectrums, we have generalized the many-body universal nature of strongly interacting fermions to include rotation by a simple rescaling of the Fermi energy and temperature. These results could be used as benchmarks in experiments to test the universal properties of strongly interacting rotating ultracold Fermi gases and may provide insight into the properties of rotating neutron stars.

H.M.Q. gratefully acknowledges the support of the ARC Centre of Excellence for Coherent X-ray Science.
[1] H. Heiselberg, Phys. Rev. A 63, 043606 (2001).

[2] J. Carlson, S.-Y. Chang, V. R. Pandharipande, and K. E. Schmidt, Phys. Rev. Lett. 91, 050401 (2003).

[3] T.-L. Ho, Phys. Rev. Lett. 92, 090402 (2004).

[4] H. Hu, P. D. Drummond, and X.-J. Liu, Nat. Phys. 3, 469 (2007).

[5] K. van Houcke, F. Werner, E. Kozik, N. Prokofév, B. Svistunov, M. J. H. Ku, A. T. Sommer, L. W. Cheuk, A. Schirotzek, and M. W. Zwierlein, Nat. Phys. 8, 366 (2012).
[6] R. K. Bhaduri, W. van Dijk, and M. V. N. Murthy, Phys. Rev. Lett. 108, 260402 (2012).

[7] K. M. O’Hara, S. L. Hemmer, M. E. Gehm, S. R. Granade, and J. E. Thomas, Science 298, 2179 (2002).

[8] J. E. Thomas, J. Kinast, and A. Turlapov, Phys. Rev. Lett. 95, 120402 (2005).

[9] G. B. Partridge, W. Li, R. I. Kamar, Y.-A. Liao, and R. G. Hulet, Science 311, 503 (2006). 
[10] J. T. Stewart, J. P. Gaebler, C. A. Regal, and D. S. Jin, Phys. Rev. Lett. 97, 220406 (2006).

[11] M. W. Zwierlein, C. H. Schunck, A. Schirotzek, and W. Ketterle, Nature (London) 442, 54 (2006).

[12] L. Luo, B. Clancy, J. Joseph, J. Kinast, and J. E. Thomas, Phys. Rev. Lett. 98, 080402 (2007).

[13] Y.-I. Shin, C. H. Schunck, A. Schirotzek, and W. Ketterle, Nature (London) 451, 689 (2008).

[14] C. Chin, R. Grimm, P. Julienne, and E. Tiesinga, Rev. Mod. Phys. 82, 1225 (2010).

[15] S. Nascimbène, N. Navon, K. J. Jiang, F. Chevy, and C. Salomon, Nature (London) 463, 1057 (2010).

[16] M. J. H. Ku, A. T. Sommer, L. W. Cheuk, and M. W. Zwierlein, Science 335, 563 (2012).

[17] R. Friedberg and T. D. Lee, Phys. Rev. B 40, 6745 (1989).

[18] K. V. Kheruntsyan and P. D. Drummond, Phys. Rev. A 61, 063816 (2000).

[19] I. Bloch, J. Dalibard, and W. Zwerger, Rev. Mod. Phys. 80, 885 (2008).

[20] M. Inguscio, W. Ketterle, and C. Salomon, Ultracold Fermi Gases (IOS Press, Amsterdam, 2008).

[21] T. Busch, B.-G. Englert, K. Rzaewski, and M. Wilkens, Found. Phys. 28, 549 (1998).

[22] F. Werner and Y. Castin, Phys. Rev. A 74, 053604 (2006).

[23] F. Werner and Y. Castin, Phys. Rev. Lett. 97, 150401 (2006).

[24] J. P. Kestner and L.-M. Duan, Phys. Rev. A 76, 033611 (2007).

[25] K. M. Daily and D. Blume, Phys. Rev. A 81, 053615 (2010).
[26] X.-J. Liu, H. Hu, and P. D. Drummond, Phys. Rev. Lett. 102, 160401 (2009).

[27] X.-J. Liu, H. Hu, and P. D. Drummond, Phys. Rev. A 82, 023619 (2010).

[28] T. Stöferle, H. Moritz, K. Günter, M. Köhl, and T. Esslinger, Phys. Rev. Lett. 96, 030401 (2006).

[29] X. Cui, Few-Body Systems 52, 65 (2012).

[30] D. Rakshit, K. M. Daily, and D. Blume, Phys. Rev. A 85, 033634 (2012).

[31] D. B. Kaplan and S. Sun, Phys. Rev. Lett. 107, 030601 (2011).

[32] B. C. Mulkerin, C. J. Bradly, H. M. Quiney, and A. M. Martin, Phys. Rev. A 85, 053636 (2012).

[33] E. C. Stoner, Proc. R. Soc. London, Ser. A 165, 372 (1938).

[34] G.-B. Jo, Y.-R. Lee, J.-H. Choi, C. A. Christensen, T. H. Kim, J. H. Thywissen, D. E. Pritchard, and W. Ketterle, Science 325, 1521 (2009).

[35] C. Sanner, E. J. Su, W. Huang, A. Keshet, J. Gillen, and W. Ketterle, Phys. Rev. Lett. 108, 240404 (2012).

[36] S.-Y. Chang, M. Randeria, and N. Trivedi, Proc. Natl. Acad. Sci. USA 108, 51 (2011).

[37] G. J. Conduit, A. G. Green, and B. D. Simons, Phys. Rev. Lett. 103, 207201 (2009).

[38] G. J. Conduit and B. D. Simons, Phys. Rev. Lett. 103, 200403 (2009).

[39] K. Huang, Statistical Mechanics, 2nd ed. (Wiley, New York, 1963). 